\title{
The relation of the phase-locked fixation saccade-linked component of alpha rhythm to change of stimulus illuminance'
}

KENNETH GAARDER, ARTHUR ALTERMAN AND WALTER KROPFL

NATIONAL INSTITUTE OF MENTAL HEALTH AND WALTER REED ARMY INSTITUTE OF RESEARCH

Systematic variation of the stimulus illuminance of a fixation target was carried out while recording fixation eye movements and EEG. The summated EEG activity following fixation saccadic eye movements was collected and it was found that the latencies of the alpha-like component of the summated activity did not vary significantly with stimulus change. The amplitude of the alpha-like activity did, however, follow the expected pattern of decreasing with increased stimulus illuminance.

It has recently been demonstrated (Gaarder et al, in press) that a component of alpha rhythm is phaselocked relative to the occurrence of fixation saccadic eye movements. This finding has been related to a model of perception involving discontinuous edge information transmission wherein each saccade presents a packet of information for short-term storage in a reverberating circuit where "bins"' are reflected by the alpha cycle.

Within the context of the finding of phase-locking and the model used to explain it, one important question arising is what effect variation of illuminance of the stimulus has upon phase-locking of the saccade-linked component of the alpha rhythm. This question will be examined here.

\section{Method}

The procedure and apparatus used have been fully described elsewhere (Gaarder et al, 1964) but will be briefly reviewed here. Two normal male Ss, ages 29 and 34 , were each run in 10 separate sessions. The EEG was recorded from bipolar electrodes on the inion and $5 \mathrm{~cm}$ anteriorly. Fixation eye movements were recorded by a standard technique and their electrical signal was fed to a special analog computer which gave trigger pulses to a Mnemotron Computer of Average Transients (CAT) whenever saccades occurred. Thus, the data generated by the experimental procedure were summated EEG activities following saccadic eye movements.

A continuously illuminated fixation target of 5 degrees overall diameter consisting of 53 circular spots 20 min. in diameter was the only stimulus. Its illuminance could be varied between runs in steps over five 1.0 $\log$ unit values from a maximum of $2 \times 10^{5}$ trolands to a minimum extimated by psychophysical means to be between 1.0 and $2.0 \mathrm{log}$ units above absolute threshold.

An experimental session consisted of one ascending and one descending series of illuminances covering all five values. Peak latency and trough latency measurements were made on the alpha-like activity between 200 and $500 \mathrm{msec}$. following saccades for each obvious peak and trough and grouped according to luminance of fixation spot.

Results

A typical set of data used for these measures is shown in Fig. 1. Notice the obvious phase locking. The bar graph in Fig. 2 shows the means and standard deviations of the peak and trough latency measures. Again the phase-locking is obvious. Since means are largely within the standard deviations of the other illuminance values and there is no obvious pattern to the variation, with the sample size used there are no significant differences between stimulus conditions. On the other hand, the range of differences between interpeak and intertrough means is $25 \mathrm{msec}$. This means that further analysis with larger sample size could conceivably show reliable differences in alpha frequencies of up to $2 \mathrm{cps}$.

The amplitude of the phase-locked alpha-like activity was strongly related to stimulus illuminance, since at the greatest illuminances it was either greatly attenuated or absent. Because of the well-known extreme variability of alpha amplitude from sample to sample this was not quantitatively studied. In general, we found these relations between fixation spot illuminance and summated

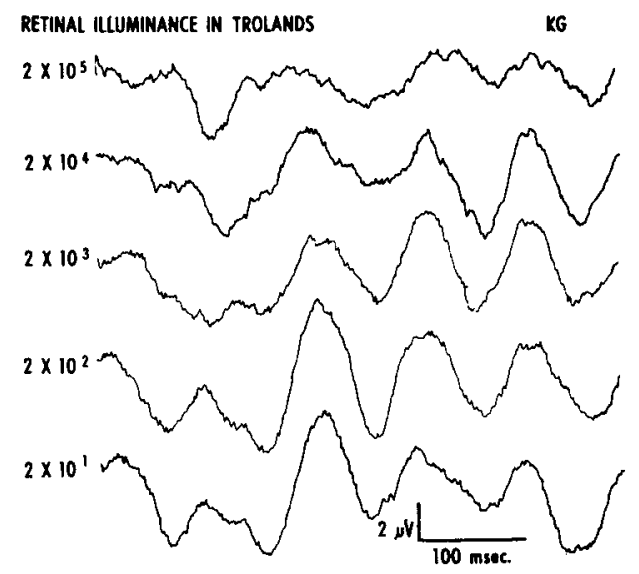

Fig. 1. Typical Set of Evoked Responses at Different Stimulus lluminance. Each run at the designated retinal illuminance. Note the phase-locking of the alpha-like waves in the last $300 \mathrm{msec}$. Compare with Fig. 2 of Gaarder et al (in press), recorded at lower illuminance, showing no typical evoked response wave form in first 200 msec. Also compare with Fig. 1 of Gaarder et al (1964) where greater illuminance blocks most of the later alpha-like activity. Inion electrode positive downward. 


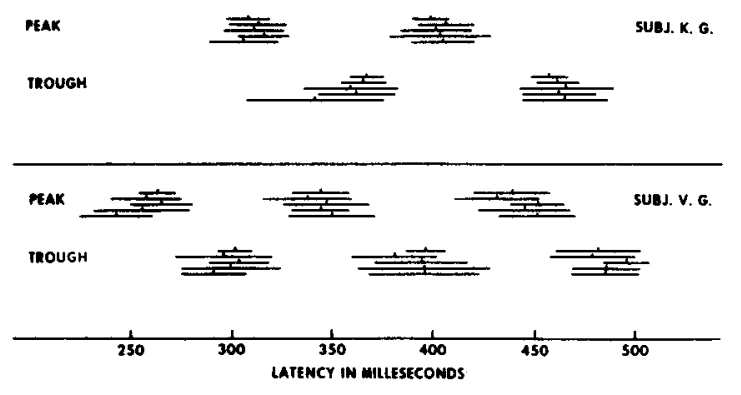

Fig. 2. Bar Graph of Peak and Trough Latencies of Alpha-Like Activity at Different Stimulus Illuminances. Each bar represents one standard deviation to left and right of the midpoint mean at a particular illuminance. Sample size for $\mathbf{S}$ KG varied between 10 and 19 peak or trough latency measurements with a median of 12.6 measurements. Sample size for S VG varied between 6 and 17 with a median of 9.6. Groups of bars represent decreasing illuminance from top to bottom $\left(2 \times 10^{5}, 2 \times 10^{4}, 2 \times 10^{3}, 2 \times 10^{1}\right.$ trolands retinal illuminance).

EEG activity: at very low illuminance the entire summated activity consisted of alpha-like activity without the detectable early evoked response of Fig. 1 (see Gaarder et al in press, Fig. 2); at slightly higher illuminance there was a typical evoked response wave form in the first $200 \mathrm{msec}$., followed by alpha-like activity (Fig. 1); at still higher illuminance both alpha in the raw EEG and alpha-like summated activity disappeared, leaving only the typical early evoked response (Gaarder et al, 1964, Fig. 1).

\section{Discussion}

Our experiment has provided a partial answer to the question of how stimulus illuminance affects the phase-locked alpha component under study. With an adequate sized sample studied no reliable differences were found in the wave-length of the alpha-like activity with change of stimulus illuminance. Thus there is no positive evidence for assuming that the timing of the alpha-like cycles is related to the stimulus illuminance. On the other hand we have not absolutely ruled out the possibility of such a relationship. It has become obvious, however, that a much larger amount of data would have to be studied to demonstrate it and that the relationship would be of an order of magnitude of several cps or less.

Regarding the amplitude of the alpha-like component we have shown that there is an inverse relation of the amplitude of the alpha-like component to the illuminance of the stimulus. This is, however, entirely consistent with the general property of alpha rhythm of attenuating with increased stimulus intensity.

The major significance of these findings is that they can be reconciled to a picture of CNS timing relationships in which the rate of gating or pacing is not heavily dependent on the intensity of the stimulus within wide limits. While it is well established that reaction times and velocity of nerve impulse conduction depend on stimulus intensity and that the pacing of alpha rhythm can be driven by the rate of stimulus presentation, it does not necessarily follow that the rate of pacing is determined by the stimulus intensity.

\section{References}

Gaarder, K., Krauskopf, J., Graf, V., Kropfl, W., \& Amington, J. C. Averaged brain activity following saccadic eye movements. Science, 1964, 146, 1481-1483.

Gaarder, K., Koresko, R. L., \& Kropfl, W. The phasic relation of a component of alpha rhythm to fixation saccadic eye movements. EEG clin. Neurophysiol., in press.

\section{Note}

1. Supported in part by PHS grant MH 06554 to the Chestnut Lodge Research Institute. 